\title{
Correction to: Which elderly newly diagnosed glioblastoma patients can benefit from radiotherapy and temozolomide? A PERNO prospective study
}

\author{
Enrico Franceschi ${ }^{1} \cdot$ Roberta Depenni $^{2} \cdot$ Alexandro Paccapelo $^{1} \cdot$ Mario Ermani $^{3} \cdot$ Marina Faedi $^{4} \cdot$

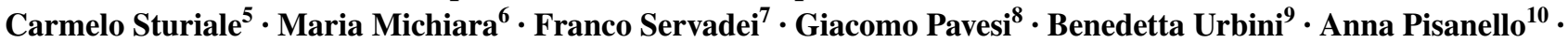 \\ Girolamo Crisi $^{11}$ - Michele A. Cavallo ${ }^{12}$ - Claudio Dazzi ${ }^{13}$ - Claudia Biasini ${ }^{14}$ Federica Bertolini $^{2}$. \\ Claudia Mucciarini $^{15}$ - Giuseppe Pasini ${ }^{16}$ - Agostino Baruzzi ${ }^{17,18}$ • Alba A. Brandes ${ }^{1}$ The PERNO Study Group
}

Published online: 9 November 2017

(C) Springer Science+Business Media, LLC 2017

\section{Correction to: J Neurooncol (2016) 128:157-162 https://doi.org/10.1007/s11060-016-2093-1}

The members of the PERNO Study Group were not individually captured in the metadata of the original publication. They are included in the metadata of this publication.

PERNO Study Group PERNO Study Group Steering committee: Baruzzi A. (Chair); Albani F., Calbucci F., D’Alessandro R., Michelucci R. (IRCCS Institute of Neurological Sciences, Bologna, Italy); Brandes A. (Department of Medical Oncology, BellariaMaggiore Hospitals, Bologna, Italy); Eusebi V. (Department of

The online version of the original article can be found under https://doi.org/10.1007/s11060-016-2093-1.

Alba A. Brandes

alba.brandes@yahoo.it

1 Department of Medical Oncology, Bellaria Hospital, Azienda USL - IRCCS Institute of Neurological Sciences, Via Altura 3, 40139 Bologna, Italy

2 Department of Oncology, Hematology and Respiratory Diseases, University Hospital of Modena, Via del Pozzo 71, 41125 Modena, Italy

3 Department of Neurosciences, Statistics and Informatics Unit, Azienda Ospedale-Universita`, Via Giustiniani 2, 35128 Padua, Italy

4 Department of Oncology and Hematology, Istituto Scientifico Romagnolo per lo Studio e la Cura dei Tumori (IRST) IRCCS, Viale Ghirotti, 286, 47521 Cesena, Italy

5 Department of Neurosurgery, Bellaria Hospital, Azienda USL - IRCCS Institute of Neurological Sciences, Bologna, Italy

6 Department of Medical Oncology, University Hospital of Parma, Via Gramsci 14, 43100 Parma, Italy

7 Department of Neurosurgery, University Hospital of Parma, Via Gramsci 14, 43100 Parma, Italy
Hematology and Oncological Sciences “L. \& A. Seragnoli', Section of Anatomic Pathology at Bellaria Hospital, Bologna, Italy); Ceruti S., Fainardi E., Tamarozzi R. (Neuroradiology Unit, Department of Neurosciences and Rehabilitation, S. Anna Hospital, Ferrara, Italy); Emiliani E. (Istituto Oncologico Romagnolo, Department of Medical Oncology, Santa Maria delle Croci Hospital, Ravenna, Italy); Cavallo M. (Division of Neurosurgery, Department of Neurosciences and Rehabilitation, S. Anna Hospital, Ferrara, Italy).Executive committee: Franceschi E., Tosoni A. (Department of Medical Oncology, Bellaria-Maggiore Hospitals, Bologna, Italy); Cavallo M. (Division of Neurosurgery, Department of Neurosciences and Rehabilitation, S. Anna Hospital, Ferrara, Italy); Fiorica F. (Department of Radiation Oncology, S. Anna Hospital, Ferrara, Italy); Valentini A. (Division of Neurosurgery, Nuovo Ospedale Civile S. Agostino-Estense, Baggiovara, Modena, Italy); Depenni R. (Department of Oncology, Policlinico di Modena, Italy); Mucciarini C. (Department of Oncology, Ramazzini

8 Department of Neurosurgery, Ospedale S. Agostino-Estense, via Giardini 1355, 41126 Modena, Italy

9 Clinical Oncology Unit, St Anna University Hospital, Corso Giovecca 203, 44121 Ferrara, Italy

10 Neurology Unit, IRCCS-Arcispedale Santa Maria Nuova, Viale Risorgimento 80, 42123 Reggio Emilia, Italy

11 Department of Neuroradiology, University Hospital of Parma, Via Gramsci 14, 43100 Parma, Italy

12 Department of Neurosurgery, St Anna University Hospital, Corso Giovecca 203, 44121 Ferrara, Italy

13 Department of Oncology and Hematology, General Hospital, Via Randi 5, 48100 Ravenna, Italy

14 Department of Oncology and Hematology, Oncology Unit, Azienda Ospedaliera Guglielmo da Saliceto, Via Taverna 49, 29100 Piacenza, Italy

15 Medical Oncology Unit, Ramazzini Hospital, Via Molinari 2, 41012 Carpi, Italy

16 Department of Medical Oncology, Infermi Hospital, Via Settembrini 2, 47900 Rimini, Italy 
Hospital, Carpi, Modena, Italy); Crisi G. (Department of Neuroradiology, Maggiore Hospital, Parma, Italy); Sasso E. (Department of Neurological Sciences, Maggiore Hospital, Parma, Italy); Biasini C., Cavanna L. (Department of Oncology and Hematology, Guglielmo da Saliceto Hospital, Piacenza, Italy); Guidetti D. (Department of Neurology, Guglielmo da Saliceto Hospital, Piacenza, Italy); Marcello N., Pisanello (A) (Department of Neurology, Istituto in tecnologie avanzate e modelli assistenziali in oncologia, IRCCS, S. Maria Nuova Hospital, Reggio Emilia, Italy); Cremonini A.M., Guiducci G. (Division of Neurosurgery, M. Bufalini Hospital, Cesena, Italy).Registry Coordination Office: de Pasqua S., Testoni S. (IRCCS Institute of Neurological Sciences, Bologna, Italy).Participants: Agati R., Ambrosetto G., Bacci A., Baldin E., Baldrati A., Barbieri E., Bartolini S., Bellavista E., Bisulli F., Bonora E., Bunkheila F., Carelli V., Crisci M., Dall'Occa P., de Biase D., Ferro S., Franceschi C., Frezza G., Grasso V., Leonardi M., Marucci G., Morandi L., Mostacci B., Palandri G., Pasini E., Pastore Trossello M., Pession A., Poggi R., Riguzzi P., Rinaldi R., Rizzi S., Romeo G., Spagnolli F., Tinuper P., Trocino C. (Bologna); Dall'Agata
M., Frattarelli M., Gentili G., Giovannini A., Iorio P., Pasquini U., Galletti G., Guidi C., Neri W., Patuelli A., Strumia S. (Forlı-Cesena); Faedi M., (IRCCS Istituto Scientifico Romagnolo per lo Studio e la Cura dei Tumori); Casmiro M., Gamboni A., Rasi F. (Faenza, RA); Cruciani G. (Lugo, RA); Cenni P., Dazzi C., Guidi A.R., Zumaglini F. (Ravenna); Amadori A., Pasini G., Pasquinelli M., Pasquini E., Polselli A., Ravasio A., Viti (B) (Rimini); Sintini M. (Cattolica, RN); Ariatti A., Bertolini F., Bigliardi G., Carpeggiani P., Cavalleri F., Meletti S., Nichelli P., Pettorelli E., Pinna G., Zunarelli E. (Modena); Artioli F., Bernardini I., Costa M., Greco G., Guerzoni R., Stucchi (C) (Carpi, MO); Iaccarino C., Ragazzi M., Rizzi R., Zuccoli G. (Istituto di Ricovero e Cura a Carattere Scientifico, Reggio Emilia); Api P., Cartei F., Colella M., Fallica E., Farneti M., Frassoldati A., Granieri E., Latini F., Monetti C., Saletti A., Schivalocchi R., Sarubbo S., Seraceni S., Tola M.R., Urbini B., Zini G. (Ferrara); Giorgi C., Montanari E. (Fidenza, PR); Cerasti D., Crafa P., Dascola I., Florindo I., Giombelli E., Mazza S., Ramponi V., Servadei F., Silini EM., Torelli P. (Parma); Immovilli P., Morelli N., Vanzo C. (Piacenza); Nobile C. (Padova).

17 IRCCS Institute of Neurological Sciences, via Altura 3, 40139 Bologna, Italy

18 Department of Biomedical and Neuromotor Sciences, University of Bologna, Via Foscolo 7, 40123 Bologna, Italy 